\title{
Effet des réserves de taillis sous futaie sur la croissance du taillis. Étude préliminaire
}

\author{
JE Bergez, A Cabanettes*, D Auclair, M Bédéneau \\ INRA, Centre de recherches d'Orléans, \\ Station de sylviculture, Ardon, 45160 Olivet, France
}

(Reçu le 18 janvier 1989 ; accepté le 26 mai 1989)

\begin{abstract}
Résumé - L'influence de la présence d'un arbre de réserve sur la croissance du taillis est étudiée dans le cas d'un peuplement de taillis sous futaie pauvre en réserve de la forêt domaniale d'Orléans (Loiret), selon un dispositif à 3 répétitions. Les réserves sont des chênes pédonculés (Quercus robur $L$ ) dominant un taillis de charme (Carpinus betulus $L$ ), àgé de 40 à 50 ans. Les principales variables prises en compte sont la surface terrière, le nombre de brins et le nombre de cépées du taillis. L'influence globale individuelle d'une réserve sur la croissance d'une cépée donnée n'est pas décelable. On a, en revanche, mis en évidence un effet dépressif significatif sur la surface terriere du taillis, particulierement à moins de $5 \mathrm{~m}$ de la réserve, ainsi qu'une influence sur le mode de répartition des cépées autour de l'arbre selon les points cardinaux. Les autres tendances observées ne sont pas significatives en intensité, mais plusieurs sont répétitives dans plus de $50 \%$ des cas. Ces premiers résultats mériteraient d'être précisés dans le cadre d'un dispositif plus grand prévoyant plusieurs espèces de taillis et une bonne connaissance de la variabilité du sol.
\end{abstract}

taillis sous futaie / croissance / surface terrière / répartition spatiale / densité de peuplement / Quercus robur / Carpinus betulus

Summary - Effect of standards on coppice growth in coppice-with-standards. Preliminary results. The influence of standards remaining for several coppice rotations, on the growth of the copice understorey, was studied in the Forêt d'Orleans (Central France). Oak standards (Quercus robur L) dominated a 40-50 year old hornbeam (Carpinus betulus $L$ ) coppice. The trial comprised 3 replicates. The main stand variables measured were basal area, number of sprouts and number of stools. No influence of a standard on individual stool growth could be shown. A significant negative effect on coppice basal area was observed. closer than $5 \mathrm{~m}$ from the standard, as well as an influence on stool distribution around the standard. Other tendencies were not statistically significant, but several of them are repetitive. These initial results need to be validated further on larger trials taking into account several coppice species with detailed information on soil variability.

\section{coppice-with-standard / growth / basal area / spatial distribution / forest density / Quercus robur / Carpinus betulus}

* Correspondance et tirés à part, à l'adresse actuelle suivante: INRA, Station de recherches forestières, Laboratoire de sylviculture et écologie, Domaine de l'Hermitage, Pierroton, 33610 Cestas. 


\section{INTRODUCTION}

Parmi les études consacrées aux relations, positives ou négatives, existant entre le taillis et les réserves du taillis sous futaie (TSF), on trouve de nombreuses références concernant l'influence du taillis sur la croissance, la forme et la production des réserves (par exemple : Bartet, 1891 ; Le Goff, 1984 ; Bisch et Auclair, 1988). Cette préoccupation majeure, qui prend d'ailleurs souvent le régime de la futaie comme modèle sylvicole de référence, peut s'expliquer par l'intérêt économique représenté par l'étage futaie du TSF. En revanche, l'influence symétrique des réserves sur la croissance et la production du taillis a été très peu étudiée. Si l'on excepte quelques rares travaux qui ont contribué à quantifier globalement le phénomène (Ranger et al, 1981 ; Auclair et Bige, 1984), la plupart des références sont de l'ordre des généralités et peuvent être contradictoires. Ainsi, Stewart (1980) mentionne le caractère "moins vigoureux" du taillis de TSF par rapport au taillis simple, et l'on admet, à l'inverse, un possible rôle positif des réserves sur le taillis, ces réserves lui fournissant un "abri climatique latéral» (Pardé, 1980).

La connaissance des conditions de croissance du taillis de TSF est pourtant un aspect complémentaire nécessaire à la connaissance objective de ce régime forestier, et peut contribuer ainsi à évacuer les interminables et violentes polémiques à son égard (Aubry et Druelle, 1987). Sur le plan économique, des études ont déjà montré que, dans les TSF pauvres en réserves et à réserves mal conformées, le taillis procure la plus grande partie du revenu économique (Triou et Vache- rat, 1986). Sur le plan de la production, on peut se demander si la présence des réserves constitue seulement un apport de bois d'œuvre ou si cet apport s'accompagne également d'une augmentation (ou d'une diminution) de la quantité de petits bois. Sur les plans méthodologique et théorique, il y a lieu de savoir si l'on peut transposer au TSF les lois de croissance établies pour le taillis simple (Pagès, 1985 ; Bédéneau, 1988) ; l'enjeu pratique en est la qualité des estimations de production de ces peuplements. Ces problématiques rejoignent celles que l'on rencontre dans d'autres formations forestières pluristratifiées (notamment en forêt tropicale) et que l'on rencontrera sans doute également dans l'avenir, en dépit de la régression du TSF classique. En effet, dans les récents essais de sylviculture traditionnelle modifiée (Teissier du Cros, 1988), qui constituent l'une des prémices de la forêt future, il y aura lieu de connaître et de maîtriser les interactions existant entre un taillis à courte rotation et une strate "futaie" d'essences précleuses plantées à grand espacement.

La nature des effets exercés par les réserves sur le taillis peut être rapprochée de celle des phénomènes de compétition entre arbres d'un même peuplement : concurrence pour la lumière, l'eau et les éléments nutritifs du sol. Mais, du fait du caractère dominant et épars de la composante réserve, il s'y ajoute une forte hétérogénéité spatiale, génératrice de microclimats diversifiés (Pardé, 1980). L'importance du houppier des réserves suggère un rôle majeur de la lumiere, dont les effets sur le développement des végétaux sont assez bien connus (Roussel, 1972, 1978). Pour traduire les différents degrés d'interception de la lumière par le houppier d'une réserve et l'influence 
de son appareil racinaire, on distingue généralement autour de celle-ci (Giordano et al, 1986) une zone dite de couvert, qui correspond à la surface de projection orthogonale de la cime sur le sol, une zone dite d'ombrage qui représente la surface totale hors couvert balayée par l'ombre de la cime au cours d'une journée ou d'une année complète et une zone d'influence racinaire (fig 1).

Compte tenu de la complexité des facteurs en cause et de la diversité des réponses possibles (taux de débourrement des bourgeons dormants, effets sur la croissance et la morphogenèse des rejets), nous avons choisi de nous limiter à des aspects préliminaires simples. L'objectif de notre étude est de mettre en évidence la présence ou l'absence d'un effet global de ces diffé-

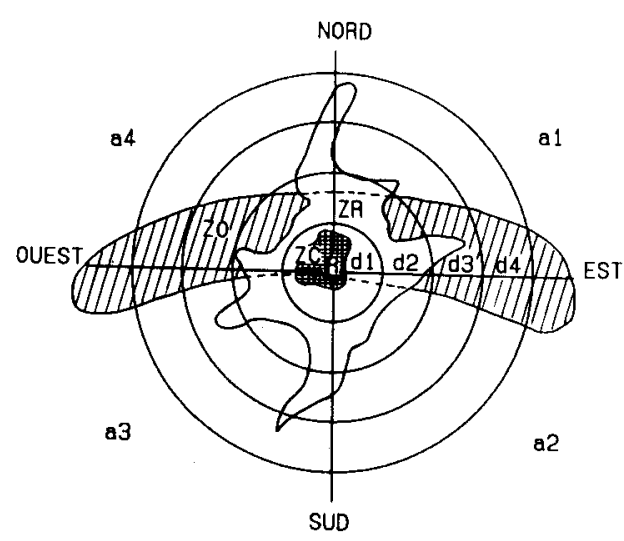

Fig 1. Schéma des différentes zones d'influence d'une réserve sur le taillis, et zonation du plan autour de la réserve utilisée pour décrire la répartition des cépées et des grandeurs dendrométriques. $\mathrm{R}=$ tronc de la réserve $; \mathrm{ZC}=$ zone de couvert $;$ $Z R=$ zone $d$ 'influence racinaire $; Z O=$ zone d'ombrage; $a_{1}, a_{2}, a_{3}, a_{4}:$ secteurs d'angles; $d_{1}$, $d_{2}, d_{3}, d_{4}$ : intervalles de distance à la réserve. rents facteurs sur la croissance du taillis à un instant donné. L'échelle d'étude choisie, qui nous a semblé la plus favorable, est celle de la réserve et de sa zone d'influence immédiate (fig 1); le site retenu est suffisamment pauvre en réserves pour permettre l'étude individuelle de leur influence sur le taillis. L'unité de travail a été, d'une part, l'individu cépée et, d'autre part, une surface élémentaire déterminée.

\section{MATÉRIEL ET MÉTHODE}

\section{Matériel}

Le site retenu est la parcelle 176 du massif de Lorris de la forêt domaniale d'Orléans (Loiret). Le peuplement est un TSF constitué d'un taillis de charme (Carpinus betulus $L$ ) relativement pur et homogène, âgé de 40 à 50 ans, et de réserves de chêne pédonculé (Quercus robur $L$ ) distantes localement d'environ $40 \mathrm{~m}$. Les espèces secondaires du taillis sont le chêne et le bouleau. Les caractéristiques dendrométriques sont résumées dans le tableau I.

Le dispositif est constitué de 3 répétitions, chacune d'elles étant une placette circulaire de $25 \mathrm{~m}$ de rayon délimitée autour d'une réserve. On a en effet considéré, selon Daniels (1976) et Lorimer (1983), que le rayon d'investigation de la réserve est proportionnel à celui de son houppier, selon un coefficient de 3,5 (Lorimer, 1983), le rayon maximum du houppier des réserves étant ici de $7 \mathrm{~m}$.

\section{Mesures}

Sur les réserves ont été mesurées les grandeurs suivantes, destinées à traduire le degré et le mode de développement de l'arbre au-dessus du sol : la hauteur totale $(\mathrm{Ht})$ et la hauteur de la premiere branche vivante (H1), mesurées au décimètre près à l'aide du relascope de Bitterlich; la circonférence du tronc à $1,30 \mathrm{~m}$, prise au ruban au millimetre pres ; la surface de la projection orthogonale du houppier (SH), selon la méthode décrite par Leguay (1982) et Pardé 
Tableau I. Principales caractéristiques dendrométriques des 3 placettes. Les biomases aériennes ligneuses de chaque réserve ont été estimées à partir des régressions établies par Bisch et Auclair (1988). Pour la signification des symboles, se reporter à l'annexe 1.

\begin{tabular}{lccc} 
& \multicolumn{3}{c}{ Répétitions } \\
& 1 & 2 & 3 \\
\hline Réserve & & & \\
Espèce & Quercus robur L & Quercus robur L & Quercus robur L \\
Ht $(\mathrm{m})$ & 18,5 & 23,5 & 25,5 \\
H1 $(\mathrm{m})$ & 8,8 & 10,3 & 10,0 \\
C130 $(\mathrm{cm})$ & 163 & 192 & 220 \\
SH $\left(\mathrm{m}^{2}\right)$ & 42,76 & 98,38 & 72,69 \\
PS total $(\mathrm{kg})$ & 2027 & 2888 & 3854 \\
PS houppier $(\mathrm{kg})$ & 586 & 1207 & 1515 \\
PS tronc $(\mathrm{kg})$ & 1441 & 1681 & 2339 \\
& & & \\
Taillis & 75,8 & 69,6 & 84,2 \\
\% charme & 296 & 579 & 165 \\
sur moyenne par cépée $\left(\mathrm{cm}^{2}\right)$ & 4,52 & 5,25 & 2,72 \\
nb moyen par cépée & 33,1 & 48,0 & 30,8 \\
cir moyenne par cépée $\left(\mathrm{cm}^{2}\right)$ & & & 26,3 \\
Somme des sur & & & \\
rapportée à l'ha $\left(\mathrm{m}^{2}\right)$ & 27,2 & & \\
\hline
\end{tabular}

et Bouchon (1988). Les coordonnées de la réserve par rapport à un point de référence ont été prises au théodolite.

Pour le taillis, l'individu choisi est la cépee, sur laquelle nous avons pris en compte 2 grandeurs classiquement sensibles à la compétition en futaie (Assmann, 1970), mais également en taillis (Trimble, 1974 ; Lamson, 1983) : la mortalité des rejets et leur croissance en diamètre. Les variables retenues sont les suivantes : circonférence du plus grand rejet, prise au millimètre près, nombre de rejets vivants, nombre de rejets morts sur pied présents lors des mesures, surface terrière de la partie vivante de la cépée. Les coordonnées du centre de chaque cépée ont été relevées au théodolite.

\section{Traitement des données}

Nous avons traité 2 types de données: d'une part, les variables relatives aux cépées considérées individuellement, en ne prenant en compte que l'espèce charme, d'autre part les variables relatives à des surfaces élémentaires déterminées, en prenant en compte soit la totalité des espèces présentes, soit le charme seul.

\section{Variables concernant l'individu cépée}

S'agissant de confronter les fluctuations des 4 variables dendrométriques définies cidessus avec la localisation des individus correspondants par rapport à la réserve, l'une des méthodes les plus adéquates est l'analyse factorielle des correspondances (AFC) (Fénelon, 1981 ; Cibois, 1983). C'est celle que nous avons retenue ici.

Une étude préalable des coefficients de corrélation partielle entre les variables brutes a été effectuée, afin de mettre en évidence les liaisons linéaires et les redondances existant entre variables. On a procédé ensuite à une standardisation des données en calculant les valeurs centrées réduites des variables au niveau de chaque répétition, de manière à traiter globalement les 3 placettes et à éliminer la variabilité inter-répétitions.

La mise en œuvre de la technique d'analyse factorielle des correspondances a nécessité tout d'abord un classement des valeurs des variables à mettre en correspon- 
dance. Les valeurs des variables dendrométriques ont été réparties en 2 classes (de part et d'autre de la moyenne), et les coordonnées des cépées ont été distribuées à la fois selon les 4 cadrans nord-est, sud-est, sud-ouest, nord-ouest (N-E, S-E, S-O, N-O), et selon 4 classes de distance à la réserve (0-5 m, 5-10 m, 10-15 m et $15-20 \mathrm{~m}$ ). Cela revient à decouper le plan horizontal autour de la réserve en secteurs d'angle emboîtés (fig 1). Nous avons ensuite construit le tableau de Burt (Bouroche et Saporta, 1980) sur lequel est effectuée l'AFC. Ce tableau est obtenu en croisant entre elles les 16 catégories de valeurs définies ci-dessus, avec en intersection le nombre d'individus correspondant. Le logiciel utilisé pour l'AFC est le programme ANCORR de la bibliotheque ADDAD (Lefonen, 1981). Outre cette analyse initiale (AFC1), nous avons effectué les 2 autres analyses suivantes:

- l'AFC2 porte sur les mêmes variables que l'AFC1, redéfinies par un découpage de l'espace autour de la réserve en 16 secteurs de même surface $\left(122,7 \mathrm{~m}^{2}\right)$;

- AFC3: les angles sont ici mis en variables supplémentaires, de manière à évaluer la contribution de cette variable à l'analyse.

\section{Variables rapportées à des surfaces élémentaires}

Les individus sont ici les 16 surfaces élémentaires, définies autour de la réserve comme ci-dessus. Chaque surface est ainsi repérée par un secteur d'angle ef un intervalle de distance à la réserve. On a utilisé également 2 types de découpage de 4 cadrans cardinaux : soit selon une progression régulière du rayon (5-10-15-20 m), soit pour obtenir des surfaces égales (rayons: 10-14,
$1-17,3-20 \mathrm{~m})$. Les variables dendrométriques retenues ont été obtenues par sommation des valeurs relatives aux cépées présentes sur la surface correspondante, rapportées à l'aire de cette surface : nombre de brins vivants, nombre de brins morts, surface terriere, nombre de cépées. Du fait de la non-normalité de distribution de ces variables, leur analyse a été effectuée a l'aide de tests non paramétriques. L'analyse globale des effets distance et angle a été réalisée à l'aide du test de Friedman (Dagnélie, 1970), qui consiste à déterminer le rang des différents traitements dans chaque bloc et à effectuer le calcul d'un $\chi^{2}$ basé sur les sommes des carrés des rangs par traitement. Un bloc est, ici, chacune des 12 intersections répétition, niveau de l'autre facteur. On a comparé individuellement les effets des differents niveaux d'un facteur à l'aide de la technique des "comparaisons multiples», dérivée du test de Friedman (Dacunha-Castelle et Tomassone, 1975).

\section{RÉSULTATS}

\section{Approche linéaire}

Le tableau II présente les coefficients de corrélation partielle calculés entre les variables brutes mesurées sur le taillis. On constate que les corrélations entre les variables dendrométriques et les variables de localisation sont faibles. Dans l'ensemble, on n'observe pas de tendance linéaire très marquée, sauf en ce qui concerne les associations de la surface terrière avec le nom-

Tableau II. Matrice des corrélations partielles entre les variables mesurées sur le taillis. Seuils de signification : 0,1037 au risque de $5 \%$ et 0,135 au risque de $1 \%$. Pour la signification des symboles des variables, se reporter à l'annexe 1.

\begin{tabular}{lrrrrrr}
\hline & $n b$ & $n m$ & cir & sur & $a$ & $d$ \\
\hline$n b$ & 1,000 & & & & & \\
$n m$ & 0,545 & 1,000 & & & & \\
cir & 0,394 & 0,232 & 1,000 & & & \\
sur & 0,799 & 0,447 & 0,700 & 1,000 & & \\
$a$ & 0,097 & 0,095 & 0,160 & 0,157 & 1,000 & \\
$d$ & $-0,062$ & $-0,040$ & $-0,108$ & $-0,150$ & $-0,015$ & 1,000 \\
\hline
\end{tabular}


bre de rejets et avec la circonférence du rejet dominant de la cépée.

\section{Analyses factorielles des correspon- dances}

Le tableau 111 , relatif à la première analyse (AFC1), donne les valeurs propres et les coordonnées des variables sur les 4 facteurs principaux d'inertie (explication de $69,6 \%$ de la variance totale). Les figures $2 a$ et $2 b$ representent respectivement les projections des variables sur les plans des facteurs 1-2 et 1-3.

On voit que le facteur 1 est très fortement expliqué par la surface terrière, ainsi que par le nombre de brins (morts ou vivants) et la circonférence du brin dominant, opposant ainsi les grosses cépées aux petites cépées. Ce point est à rapprocher de la liaison linéaire mise en évidence précédemment entre ces variables.

Les facteurs 2 et 3 operent une opposition entre secteurs angulaires $\mathrm{N}-\mathrm{E}$ par rapport à $\mathrm{S}-\mathrm{E}$ pour le facteur 2 , et $\mathrm{N}-\mathrm{E}$ par rapport à $\mathrm{N}-\mathrm{O}$ pour le facteur 3. Mais les variables dendrométriques sont pratiquement orthogonales aux facteurs 2 et 3 , ce qui écarte toute possibilité d'une répartition particulière des cépées selon les secteurs d'angle.

En ce qui concerne les distances, on observe une tendance à leur répartition le long du premier axe (fig $2 a$ et $2 b$ ), qui pourrait indiquer un effet de la distance à la réserve sur l'augmentation de la surface terriere et du nombre de brins vivants. II faut toutefois rester prudent, car d'une part la qualité de représentation des distances sur les 2 plans 1-2 et 1-3 est faible (tableau III), et d'autre part les distances sont bien représentées sur des axes factoriels plus lointains, pour lesquels la contribution des variables dendrométriques est très faible.

Les ACF2 et 3 ne modifient pratiquement pas les résultats de l'AFC1: les valeurs propres, la contribution des variables aux facteurs et les coordonnées des variables sont presque inchangées ; les angles sont orthogonaux aux autres données.
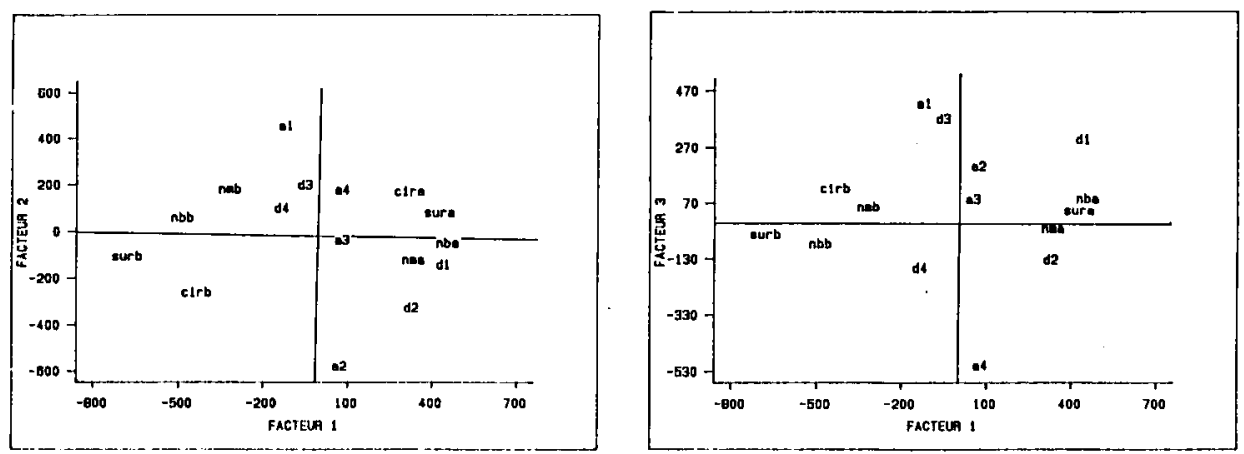

Fig $2 \mathbf{a}$ et b. Représentation des variables résultant de l'analyse factorielle des correspondances AFC1 La signification des symboles des variables est indiquée en annexe 1. a. Représentation sur le plan factoriel 1-2. b. Représentation sur le plan factoriel 1-3. La variable cira est confondue avec la variable nma. 
Tableau III. Résultats de l'analyse factorielle des correspondances AFC1. QLT = qualité de représentation; $\mathbb{I N R}=\%$ d'inertie totale ; $C O R=$ corrélation au facteur ; $C T R=$ contribution à l'axe.

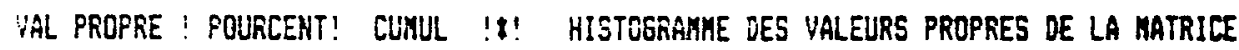
0.12978
33.467

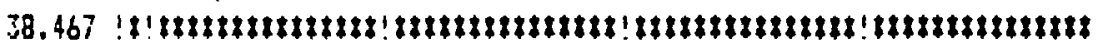
0.04033
11.969

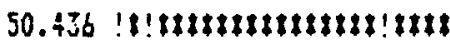
$0.03297 ! 9.773 ! 50.209$ !1!14141114114\$218!

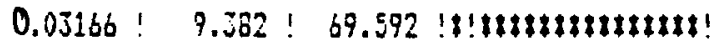

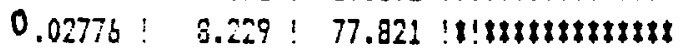
$0.02478 !+7.344 ! 35.165$ !1!11411481414
$0.02015 ! 5.766 ! 91.131$ !1!111121114
$0.01573 ! 4.663$ ! 95.794 :1!1111144
$0.01209 ! 3.583 \div 99.377$ !1!181814
$0.00210 \div 0.623 ! 100.000 ! 1 ! 1$
$0.00000 \div 0.000 \div 100.000 ! 1 !$
$0.00000 ! 0.000 ! 100.000 ! 1 !$
$0.00000 ! 0.000 ! 100.000 ! 1 !$
$0.00000 \div 0.000 \div 100.000 \div 1 !$
$0.00000 ! 0.000 ! 109.000 ! 1 !$

11 QLT POIDINR 1\#F CORCTR 2\#F CORCTR 3\#F CORCTR 4\#F CORCTR

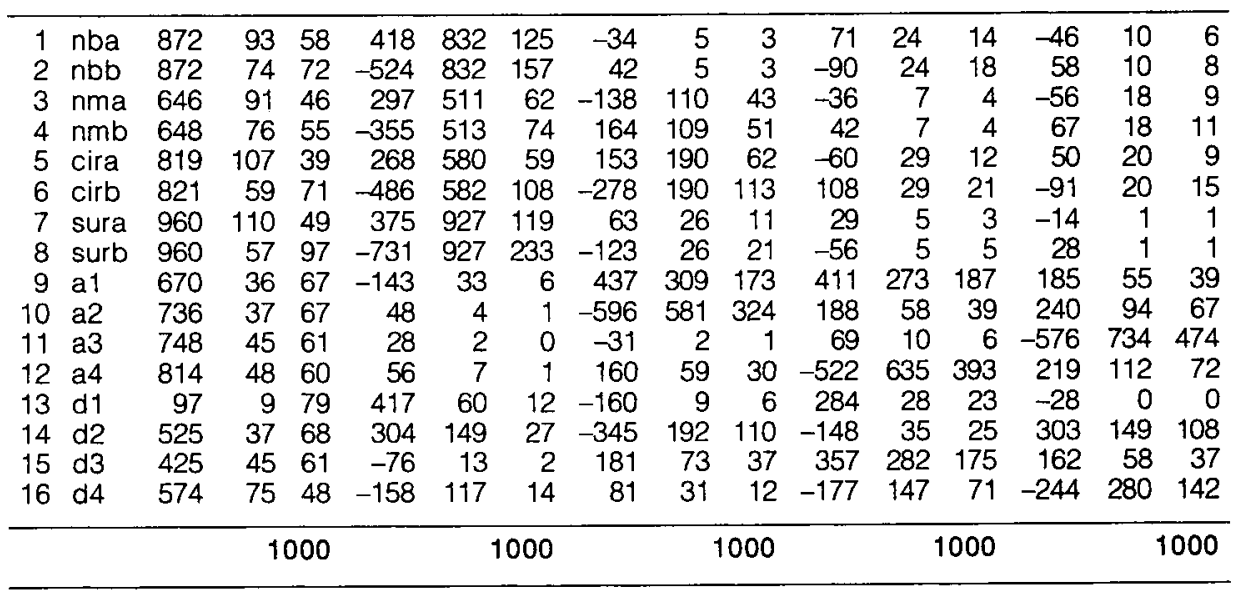




\section{Analyses des variables rapportées à la surface}

Les 2 résultats marquants sont (fig 3 , tableau IV) :

- le caractère hautement significatif $(P<0,005)$ de l'effet de la distance à la réserve sur la surface terrière, dans le seul cas d'aires inégales et pour l'ensemble des espèces du taillis. Ce résultat s'accompagne d'une différence significative entre les surfaces terrières situées, d'une part, à moins de $5 \mathrm{~m}$ et, d'autre part, à plus de $10 \mathrm{~m}$ de la réserve ;

- un effet à la limite de signification (P \# 0,05) de l'angle sur la densité d'ensouchement, dans le seul cas de surfaces égales. Mais on ne note pas de différences significatives individuelles entre les secteurs d'angle, ce résultat global n'étant vérifié que dans 10 cas sur 12 à proximité de la réserve. Au-delà de $10 \mathrm{~m}$, le nombre de cépées présentes est toutefois toujours plus élevé à l'ouest qu'à l'est.

En dehors de ces 2 résultats, on n'observe aucun effet significatif des facteurs. Il existe néanmoins des tendances, dénotées par une répétition de résultats partiels (fig 3 ) :

- la densité de brins vivants est moindre à moins de $5 \mathrm{~m}$ qu'au-delà dans 8 cas sur 12 ( 5 cas sur 6 au nord) ; en
DENSITE DE BAINS

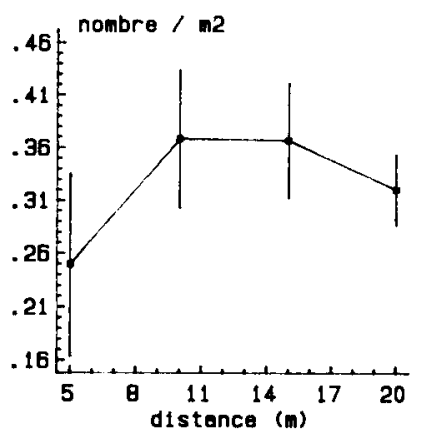

DENSITE DE BRINS

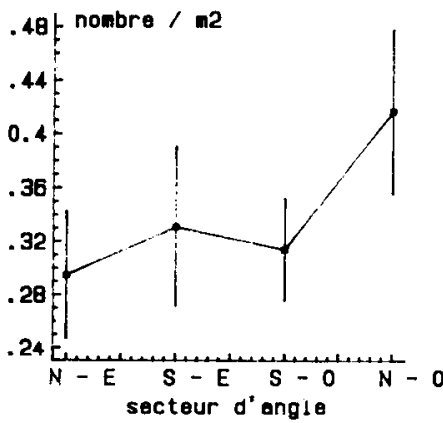

SURFACE TEARIERE

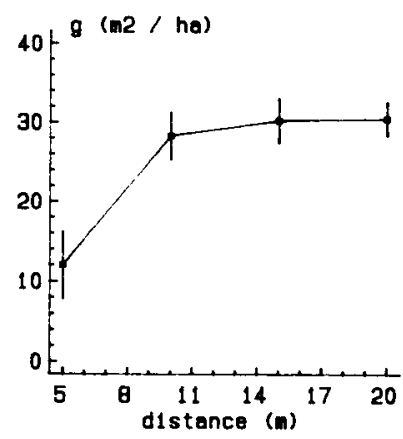

MORTALITE

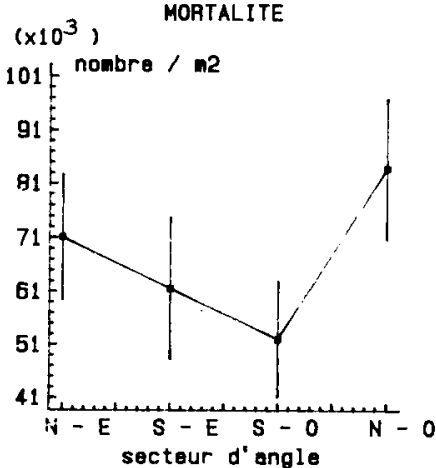

ENSOUCHEMENT

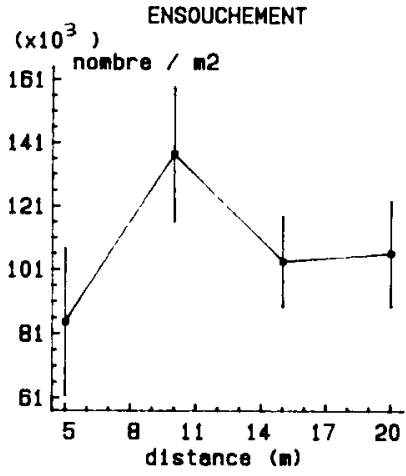

ENSOUCHEMENT

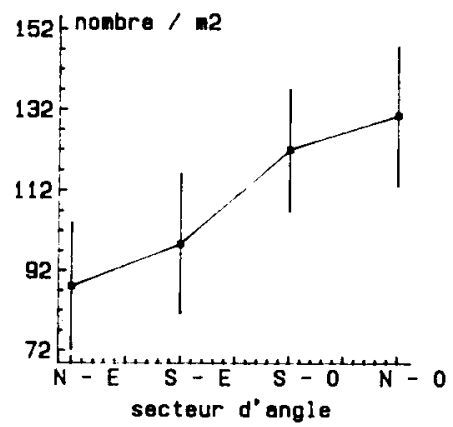

Fig 3. Résultats significatifs et principales tendances dans les fluctuations des variables dendrométriques du taillis selon la distance (en haut) ou l'orientation (en bas) par rapport aux réserves. Les points reliés sont les valeurs moyennes, les barres verticales les erreurs standard. 
Tableau IV. Résultats du test de Friedman pour les 4 variables et les 2 facteurs étudiés. La valeur du $\chi^{2}$ obtenue est comparée à un $\chi^{2}$ thérique à 3 degrés de liberté. P est l'intervalle de probabilité où se situe celle d'obtenir un $\chi^{2}$ de valeur superieure au $\chi^{2}$ observé. Les données sont issues de surfaces égales pour les comparaisons portant sur le facteur angle. et de surfaces inégales pour les comparaisons portant sur le facteur distance.

\begin{tabular}{llcc}
\hline \multicolumn{1}{c}{ Variable } & \multicolumn{1}{c}{ Facteur } & $\chi^{2}$ observé & $P$ \\
\hline Surface terrière & Distance & 12,8 & $<0,005$ \\
& Angle & 3,3 & $0,10-0,25$ \\
Densité de brins vivants & Distance & 4,9 & $0,25-0,50$ \\
& Angle & 3,3 & $0,10-0,25$ \\
Densité de brins morts & Distance & 5,2 & $0,10-0,25$ \\
& Angle & 3,4 & $0,10-0,25$ \\
Densité d'ensouchement & Distance & 4,2 & $0,10-0,25$ \\
& Angle & 7,3 & $0,05-0,10$ \\
\hline
\end{tabular}

exposition nord, cette grandeur est supérieure dans 8 cas sur 12 dans le secteur ouest par rapport au secteur est ; - la densité de brins morts est globalement plus élevée au nord qu'au sud ( 8 cas sur 12);

- pour la densité d'ensouchement, on constate que, dans la zone $0-15 \mathrm{~m}$, cette grandeur est la plus élevée dans la tranche $5-10 \mathrm{~m}$ dans 9 cas sur 12

\section{DISCUSSION}

Les résultats fournis par l'AFC présentent des valeurs propres élevées (tableau III) dues à la faible variabilité inter-répétitions des données, que l'on peut attribuer au petit nombre de ces répétitions. Dans l'ensemble, aucune liaison nette n'apparaît entre les variables de localisation et les variables dendrométriques des cépées. On notera toutefois la tendance à une liaison entre distance et surface terrière, et particulièrement la discontinuité importante entre les classes $5-10 \mathrm{~m}$ et 10 $15 \mathrm{~m}$ (fig 2), qui pourrait rendre compte d'un effet du couvert plus marqué que celui de l'ombrage (le rayon maximal des houppiers est de $7 \mathrm{~m}$ ). La répartition des variables dendrométriques selon les facteurs 2 et 3 pourrait également suggérer une croissance un peu meilleure au sud, particulièrement $S-E$, par rapport au nord, particulièrement N-E. II s'agirait donc là d'un effet de l'ombrage (relativement indépendant de l'effet distance). l'AFC3 confirme toutefois le caractère très faible de cet effet. Le découpage en surfaces égales (AFC2) n'apportant pas de modifications majeures montre le faible rôle des distances; on retrouve la discontinuité dans l'effet des distances à la réserve au même niveau, plus exactement ici entre la classe 0 $10 \mathrm{~m}$ et les classes $>10 \mathrm{~m}$.

L'analyse des données rapportées à l'unité de surface a permis de mettre en évidence des effets significatifs de la réserve sur la croissance du taillis. Par rapport aux résultats moins nets fournis par l'AFC, la différence semble davantage liée à la nature des grandeurs mesurées qu'à l'efficacité de la technique d'analyse. En effet, les variables rapportées à l'unité de surface intègrent à la fois les fluctuations des variables relatives aux cépées et celles de la densité d'ensouchement. Prises individuellement, les variables "cépée" ne sont pas liées très fortement aux facteurs distance et angle, mais leur combinaison multiplicative avec la densité peut devenir significativement liée 
à ces facteurs. L'effet positif significatif de la distance à la réserve sur la seule surface terrière montre qu'il y a addition des effets sur la croissance en diamètre des brins à ceux, relativement faibles, qui agissent sur le nombre de brins et le nombre de cépees.

Le rôle significatif de l'orientation de la cépée vis-à-vis de la réserve sur la densité d'ensouchement est plus difficile à interpréter. II n'est pas lié aux conditions particulières à une répétition, comme par exemple une hétérogénéité édaphique. On peut donc penser plutôt à un effet thermique (temperatures maximales plus élevées à l'ouest qu'à l'est) ou de vent dominant (les répétitions sont situees sur une même parcelle forestière). Le faible nombre de répétitions ne permet pas de conclure avec certitude sur ce point. En ce qui concerne le nombre de brins vivants, il semble bien que cette grandeur soit plus faible en conditions d'éclairement limitantes (exposition nord à moins de $5 \mathrm{~m}$ de la réserve). Cette observation rejoint celles de Roussel (1978) et de Ducrey (1988) sur chêne vert. Enfin, la légère augmentation de l'ensouchement entre 5 et $10 \mathrm{~m}$ demanderait confirmation par d'autres études. Il pourrait s'agir d'un effet bénéfique dit d' «abri» que l'on croit remarquer souvent dans certains peuplements de TSF.

\section{CONCLUSION}

On a constaté des phénomènes de réduction de biomasse sur pied d'un taillis de TSF et d'anisotropie dans la répartition de ses cépées, qui semblent bien pouvoir être mis en relation avec la présence des réserves:

- la surface terriere du taillis a moins de $5 \mathrm{~m}$ de la réserve est en moyenne $<60 \%$ à sa valeur au-delà de $10 \mathrm{~m}$ (fig 3). Il pourrait s'agir là du rôle prédominant de la zone de couvert sur celui de la zone d'ombrage, sans que l'on puisse conclure sur la part relative des effets en cause (interception lumineuse et activité racinaire). Ce résultat suggère donc une possible limitation importante dans l'espace de l'influence dépressive de la réserve sur le taillis. Du fait des fluctuations élevées de la biomasse sur pied du taillis par unité de surface en fonction de la distance à la réserve, on ne peut délimiter avec précision une zone d'influence des reserves. II n'est en particulier pas possible ici de mettre en évidence un éventuel "rattrapage" de la perte de biomasse au-delà du houppier de la réserve ;

- une hétérogénéité dans la répartition (donc dans l'installation et la survie) des cépées autour de la réserve : augmentation de l'ensouchement de 11 à $75 \%$ en exposition ouest par rapport a l'exposition est ; là encore, il est difficile de conclure sur les facteurs en cause (effet climatique direct par les maxima thermiques, ou effet indirect par une répartition hétérogène des racines de la réserve liée au sol ou aux vents dominants).

D'autres tendances mériteraient d'être confirmées par des études plus fines effectuees en conditions mieux contrôlées. La mise au point d'un indice de compétition pour le TSF, prenant en compte les dimensions des réserves et des cépées ainsi que leurs distances respectives, pourrait peutêtre donner des résultats intéressants comme il en a été obtenu par différents auteurs pour des arbres de futaie (Bella, 1971 ; Ottorini, 1978 ; Brand, 1986).

Ces résultats sont bien sûr à relativiser, pour différentes raisons. Tout d'abord, l'âge élevé du taillis (40 à 50 ans) n'a pas permis d'analyser la forte concurrence qui existe au stade juvénile, avec notamment des effets morphogénétiques importants liés à la compétition pour la lumière (Roussel, 1972). Les phénomènes de compéti- 
tion, antérieurs aux observations, ne sont que partiellement pris en compte par les mesures effectuées. Les essences étudiées ici sont particulières ; le charme est une espèce de demi-ombre (Roussel, 1972) et possède à ce titre un point de compensation relativement bas, ce qui pourrait limiter sa sensibilité à l'interception de la lumière par le houppier de la réserve. On n'a considéré ici que les effets individuels d'une seule réserve à la fois alors que, les zones d'influence de ces arbres étant généralement nondisjointes dans un peuplement, leurs effets peuvent donc s'additionner localement et se montrer plus importants que dans notre cas. Enfin, l'importante variabilité intra-répétitions des données n'a pu être suffisamment réduite compte tenu du petit nombre de répétitions et de l'absence de connaissances sur la microhétérogénéité corrélative du sol.

II n'est donc pas possible ici de formuler d'hypothèses suffisamment étayees sur les mécanismes en jeu dans les interrelations entre réserve et taillis. On se contentera de suggerer quelques perspectives possibles pour de futures recherches. L'étude de taillis plus jeunes (ou l'analyse de tige de taillis plus âgés, à cernes bien individualisés) composés d'essences de sensibilités lumineuses différentes serait sans doute très prometteuse. II y aurait lieu d'ajouter la mesure de la longueur des rejets, qui n'a pas été prise en compte ici, ainsi que les phénomènes de ramification, qui peuvent influer de manière significative sur la quantité de biomasse et donc sur la production du taillis. Enfin, une synthèse de ces résultats avec les études de croissance et de production des réserves de TSF (Leguay, 1982 ; Bish et Auclair, 1988) permettrait d'avoir une vue complète et objective de ce régime sylvicole.

\section{REMERCIEMENTS}

Nous remercions l'ONF, et plus spécialement le personnel de la Subdivision de Lorris du Centre d'Orléans, sur le domaine duquel a été réalisée cette étude. Le travail technique a été mené à bien grâce à l'équipe technique de la Station de sylviculture et à celle de l'Unité d'experimentation de la biomasse forestière d'Orléans. Que Michèle Audoux soit ici remerciée pour son aide efficace dans les recherches bibliographiques. Mile Ligouzat a participé par ses judicieux conseils à l'analyse des données par l'analyse factorielle des correspondances.

\section{RÉFÉRENCES}

Assmann E (1970) The Principles of Forest Yield Study. Pergamon Press, Oxford, $506 \mathrm{p}$

Aubry S, Druelle P (1987) Histoire du taillissous-futaie ou La résurrection d'un amort qui reste à tuer». Essai bibliographique et critique, Domaine des Barres, Nogentsur-Vernisson (France), $70 p$

Auclair D, Bigé MC (1984) Une methode d'évaluation régionale des biomasses de taillis à partir des donnees de l'Inventaire Forestier National. Application à la région Centre. Ann Sci For 41, 405-426

Bartet E (1891) Recherches sur le mode d'accroissement des chênes de taillissous-futaie. Rev Eaux Forêts 30, 441-456

Bédéneau M (1988) Croissance du taillis de châtaignier en France : premiers résultats. Ann Sci For 45, 265-274

Belia IE. (1971) A new competition model for individual trees. For Sci 17, 364-372

Bisch JL, Auclair D (1988) Influence of the sylvicultural treatment (high forest or coppice with standards) on oak aboveground biomass distribution in Central France. Forestry 61, 205-217

Bouroche JM, Saporta G (1980) L'analyse des données. Collection "Que sais-je ?" $n^{\circ}$ 1854, Presses Universitaires de France, Paris, $127 \mathrm{p}$

Brand DG (1986) A competition index for predicting the vigour of planted Douglasfir in southwestern British Columbia. Can $J$ For Res 16, 23-29

Cibois P (1983) L'Analyse factorielle. Collection "Que sais-je ?" $n^{\circ}$ 2095, Presses Universitaires de France, Paris, $127 p$ 
Dacunha-Castelle D. Tomassone R (1975) Élements de statistique non paramétrique. INRA, Laboratoire de biométrie du CNRZ, Jouy-en-Josas (France), Doc 75/09, sp

Dagnélie P (1970) Thérie et méthodes statistiques. Applications agronomiques, vol 2. Duculot, Gembloux (Belgique), $451 \mathrm{p}$

Daniels RF (1976) Simple competition indices and their correlation with annual 10 blolly pine tree growth. For Sci 22, 454-456

David A (1979) Les peuplements de taillis de la basse forêt de Paimpont. Étude dendrologique et écologique. Mémoire de $3^{e}$ année de l'École Nationale des Ingénieurs des Travaux des Eaux et Forêts, Nogent-sur-Vernisson (France), $64 \mathrm{p}$

Ducrey M (1988) Sylviculture des taillis de chêne vert. Pratiques traditionnelles et problématique des recherches récentes. Rev For Fr 40, 302-313

Fenelon JP (1981) Qu'est-ce que l'analyse des données ? Lefonen, Paris, $311 p$

Giordano $E$, Leone A, Ragno D, Schirone B (1986) Studio dell'ombra proiettata durante l'anno dalla chioma di un albero. Un modello per il Cerro (Quercus cerris L). Italia Forestale e Montana 41, 129-150

Hubert M (1987) Le taillis, auxiliaire du reboiseur. Forêt Entreprise 46, 36-45

Lamson NI (1983) Precommercial thinning increases diameter growth of Appalachian hardwood stump sprouts. $J$ App For 93-97

Leguay $F$ (1982) Étude de la répartition de la biomasse dans les reserves de taillis sous futaie de chênes. INRA, Station de recherches sur la forêt et l'environnement, Orléans (France), Doc 82/36, 81 p

Le Goff N (1986) Indice de productivité des taillis sous futaie de chênes dans la région Centre. Ann Sci For 41, 1-34

Lorimer CG (1983) Tests of age-independent competition indices for individual trees in natural hardwood stands. For Ecol Manage $6,343-360$

Ottorini JM (1978) Aspects de la notion de densité et de croissance des arbres en peuplement. Ann Sci for 35 , 299-320

Pagès L (1986) Lois de croissance en biomasse du taillis : le robinier dans le Va!de-Loire. Ann Sci For 43, 533-550

Pardé J (1980) inconvénients et avantages des régimes de taillis du taillis sous futaie et de la futaie du point de vue écologique. La Forêt Privée 133, 19-29

Pardé J, Bouchon J (1988) Dendrométrie. École Nationale du Génie Rural, des Eaux et des Forêts, Nancy (France), $328 p$

Ranger J, Nys C, Ranger D (1981) Étucie comparative de deux écosystemes forestiers feuillus et résineux des Ardennes primaires françaises. Ann Sci for 38 259-282

Roussel L (1972) Photologie forestière. Masson, Paris, $144 \mathrm{p}$

Roussel L (1978) Lumière, gourmands et rejets de souche. Rev For Fr 30, 186-200

Stewart PJ (1980) Coppice with standards: a system for the future. Commonw For Rev 59, 149-154

Tessier du Cros E (1987) Genetic improvement and modified conventional culture of forest trees for short term biomass production. CEE contract number EN3B$0041-F$, 5th intermediate report, May $1988,5 \mathrm{p}$

Trimble GR (1974) Response to crop-tree release by 7 -year-old stems of red maple stump sprouts and northern oak advance reproduction. US For Serv Res Paper NE$303,6 \mathrm{p}$

Triou A, Vacherat E (1986) Étude économique de la conversion en région Centre. Mémoire ENITEF

Annexe 1. Signification des abréviations utilisées.

$\mathrm{Ht}$ : hauteur totale de la réserve; $\mathrm{H}_{1}$ : hauteur de la première branche vivante de la réserve : $C_{130}$ : circontérence à $1,30 \mathrm{~m}$ de la réserve: $\mathrm{SH}$ : surface de projection du houppier de la réserve: PS : poids de matière sèche; nb: nombre de brins vivants par cépée; nm : nombre de brins morts par cépée ; cir : circonférence du brin dominant de la cépée : sur : surface terrière de la cépée ; xa : valeurs de la variable $x$ inférieures à la moyenne de $x ; x b$ : valeur de la variable $x$ supérieure à la moyenne de $x ; a_{1}, a_{2}, a_{3}, a_{4}$ : désignent respectivement les secteurs angulaires nord-est, sud-est, sud-ouest et nord-ouest ; $d_{1}, d_{2}, d_{3}, d_{4}$ : intervalles de distances croissantes à la réserve. 